\title{
Substance Abuse
}

\section{Primary Care Quality and Addiction Severity: A Prospective Cohort Study}

\author{
Theresa W. Kim, Jeffrey H. Samet, Debbie M. Cheng, \\ Michael R. Winter, Dana Gelb Safran, and Richard Saitz
}

\begin{abstract}
Background. Alcohol and drug use disorders are chronic diseases that require ongoing management of physical, psychiatric, and social consequences. While specific addiction-focused interventions in primary care are efficacious, the influence of overall primary care quality (PCQ) on addiction outcomes has not been studied. The aim of this study was to prospectively examine if higher PCQ is associated with lower addiction severity among patients with substance use disorders.
\end{abstract}

Study Population. Subjects with alcohol, cocaine, and/or heroin use disorders who initiated primary care after being discharged from an urban residential detoxification program.

Measurements. We used the Primary Care Assessment Survey (PCAS), a well-validated, patient-completed survey that measures defining attributes of primary care named by the Institute of Medicine. Nine summary scales cover two broad areas of PCQ: the patient-physician relationship (communication, interpersonal treatment, thoroughness of the physical exam, whole-person knowledge, preventive counseling, and trust) and structural/organizational features of care (organizational access, financial access, and visit-based continuity). Each of the three addiction outcomes (alcohol addiction severity (ASI-alc), drug addiction severity (ASI-drug), and any drug or heavy alcohol use) were derived from the Addiction Severity Index and assessed 6-18 months after PCAS administration. Separate longitudinal regression models included a single PCAS scale as the main predictor variable as well as variables known to be associated with addiction outcomes.

Main Results. Eight of the nine PCAS scales were associated with lower alcohol addiction severity at follow-up $(p \leq .05)$. Two measures of relationship quality (communication and whole- person knowledge of the patient) were associated with the largest decreases in ASI-alc $(-0.06)$. More whole-person knowledge, organizational access, and visit-based continuity predicted lower drug addiction severity (ASI-drug: - 0.02). Two PCAS scales (trust and whole-person knowledge of the patient) were associated with lower likelihood of subsequent substance use (adjusted odds ratio, $[\mathrm{AOR}]=0.76$, 95 percent confidence interval $[95 \% \mathrm{CI}]=0.60,0.96$ and $\mathrm{AOR}=0.66,95$ percent $\mathrm{CI}=0.52,0.85$, respectively).

Conclusion. Core features of PCQ, particularly those reflecting the quality of the physician-patient relationship, were associated with positive addiction outcomes. Our findings suggest that the provision of patient-centered, comprehensive care from 
a primary care clinician may be an important treatment component for substance use disorders.

Key Words. Substance abuse, primary care, quality of care, physician-patient relationship

Primary care is being asked to expand its role in the identification and management of addictive disorders (National Council for Community Behaviorial Healthcare 2003; New Freedom Commission on Mental Health 2003). Training sessions for primary care physicians and clinical reminders have been developed to increase the use of efficacious counseling tools (Saitz et al. 2003; Saitz, Sullivan, and Samet 2000) and practical screening instruments to identify addiction disorders (Maisto and Saitz 2003). Pharmacological therapies are now available in primary care for the treatment of opioid dependence (e.g., buprenorphine) (Fiellin and O'Connor 2002) and alcohol use disorders (e.g., naltrexone and acamprosate). Various forms of integration between primary care and addictions treatment have been demonstrated to be associated with better addiction outcomes (Willenbring and Olson 1999; Weisner et al. 2001; Friedmann et al. 2003).

While there is substantial literature promoting greater access to primary care for individuals with addictions, little is known about whether the quality of primary care should be considered. In other chronic disorders such as diabetes mellitus (Campbell, Roland, and Wilkin 2001), depression (O'Malley, Forrest and Miranda 2003), and tobacco use (Safran et al. 1998), primary care quality (PCQ) is an important determinant of outcomes. The quality of primary care delivered to individuals with substance use disorders may be important for addiction outcomes as well.

How might higher quality primary care lead to better addiction outcomes? The Institute of Medicine (IOM) and others (Institute of Medicine

Address correspondence to Theresa W. Kim, M.D., Clinical Addiction Research and Education (CARE) Unit, Section General Internal Medicine, 91 E. Concord Street, Suite 200, Boston University Medical Center, Boston, MA 02118. Jeffrey H. Samet, M.A., M.D., M.P.H., Debbie M.Cheng, Sc.D., and Richard Saitz, M.D., M.P.H., are with the Clinical Addiction Research and Education (CARE) Unit, Boston University Medical Center, Boston, MA. Dr. Samet is also with Department of Social and Behavioral Sciences, Boston University School of Public Health. Dr. Cheng is also with the Department of Biostatistics, Boston University School of Public Health. Michael R. Winter, M.P.H., is with the Data Coordinating Center, Boston, MA. Dana Gelb Safran, Sc.D., is with The Health Institute, Institute for Clinical Research and Health Policy Studies, Boston, MA. 
1996; Starfield et al. 1998; Safran 2003; Kroenke 2004) define primary care as "sustained partnerships" with essential attributes of comprehensiveness, coordination, and accessibility. Alcohol and drug problems, often unrecognized in medical encounters (Saitz et al. 1997; Weisner and Matzger 2003), may be missed less frequently in comprehensive and supportive primary care relationships. Adherence to treatment recommendations for addictive disorders may be higher in the presence of such relationships between patients and their primary care physicians. As alcohol and drug dependence are often chronic illnesses with relapsing and remitting courses (McLellan et al. 2000), effective chronic disease management strategies to enhance treatment adherence and monitor disease progression may ameliorate addiction severity. Additionally, coordination of addiction, medical, psychiatric, and social services care by primary care physicians may be especially valuable for patients with comorbid physical and/or psychiatric illnesses (Johnson et al. 1995; Mertens et al. 2003; De, Samet, and Saitz 2004).

We used data from the Health Evaluation and Linkage to Primary care (HELP) study, a randomized trial of an intervention to link adults entering an urban detoxification unit to primary care. Previous analyses showed that randomization to the intervention group was associated with greater likelihood of initiating primary care but was not associated with better addiction outcomes (Samet et al. 2003). An additional analysis demonstrated that attending at least two primary care visits, regardless of randomization arm, was associated with lower addiction severity and risk of subsequent substance use (Saitz et al. 2005). The current analysis takes this line of examination a step further and asks, among individuals with addictions who attend primary care, is the quality of primary care associated with addiction outcomes, and if so, which attributes of PCQ are important?

\section{METHODS}

\section{Study Design}

This is a prospective cohort study of patients with substance use disorders who initiated primary care during the HELP study (Samet et al. 2003). People eligible for the HELP study were adult inpatients of a residential detoxification unit who spoke Spanish or English, reported alcohol, heroin, or cocaine as their first or second drug of choice, and either resided in proximity to the primary care clinic to which they would be referred, or were homeless. Patients were excluded from the parent study if they intended to continue 
existing primary care relationships, suffered significant dementia, had specific plans to leave the Boston area that would prevent research participation, could not provide contact information for tracking purposes, or were pregnant. All participants were randomly assigned to receive either standard medical care referral by clinical addictions staff on an as needed basis (usual care) or enhanced referral (the trial intervention) to primary care in the Boston area and surrounding communities. Enhanced referral included a multidisciplinary assessment (conducted in the detoxification unit at study entry), referral to a particular physician based upon patient preference, letter of introduction to the primary care physician, and follow-up phone calls to promote adherence to the initial primary care appointment (which occurred after discharge from the detoxification unit).

After the baseline research assessment at the detoxification unit, biannual research interviews were conducted over the 24-month study period. Except for demographics, all assessments, including measures of addiction severity and health care utilization, were repeated at each interview. Alcohol breath tests were performed at follow-up interviews to encourage honest reporting (Welte et al. 1998).

The current study included HELP participants who initiated primary care, regardless of randomization group, and then completed at least one follow-up interview after initiating primary care. In total, participants in the current study completed at least three separate research interviews: (1) an assessment at the detoxification unit where study recruitment took place (i.e., the "baseline" interview); (2) a subsequent interview in which the participant reported on the quality of their primary care relationship (the "PCQ assessment" interview); and (3) a follow-up interview in which addiction outcomes were assessed (the "follow-up addiction" interview).

The Institutional Review Board of Boston University Medical Center approved this study. Additional privacy protection was secured by the issuance of a Certificate of Confidentiality by the Department of Health and Human Services.

\section{Main Independent Variable}

The Primary Care Assessment Survey (PCAS; Safran et al. 1998) is a wellvalidated, patient-completed survey that measures defining attributes of primary care named by the IOM and others (Institute of Medicine 1996; Starfield et al. 1998; Safran 2003; Kroenke 2004). The PCAS is a well-validated measure of PCQ that has been used to monitor the performance of primary care 
Table 1: Summary of Item Content for Primary Care Assessment Survey Scales

\begin{tabular}{|c|c|}
\hline Defining Characteristic & Description \\
\hline \multicolumn{2}{|c|}{ Physician-patient interaction } \\
\hline Communication & $\begin{array}{l}\text { Thoroughness of primary physician's questions about symptoms, } \\
\text { attention to what patient says, clarity of explanations and } \\
\text { instructions, and advice and help in making decisions about care }\end{array}$ \\
\hline $\begin{array}{l}\text { Interpersonal } \\
\text { treatment }\end{array}$ & $\begin{array}{l}\text { Primary physician's patience, friendliness, caring, respect, and } \\
\text { time spent with patient }\end{array}$ \\
\hline $\begin{array}{l}\text { Thoroughness of } \\
\text { physical exam }\end{array}$ & Primary physician's physical examination thoroughness \\
\hline $\begin{array}{l}\text { Whole-person } \\
\text { knowledge }\end{array}$ & $\begin{array}{l}\text { Primary physician's knowledge of patient's medical history; } \\
\text { responsibilities at work, home, school; health concerns, values, } \\
\text { and beliefs }\end{array}$ \\
\hline Preventive counseling & $\begin{array}{l}\text { Whether physician has discussed the following with patient: } \\
\text { smoking, alcohol use, seatbelt use, diet, exercise, stress, safe sex }\end{array}$ \\
\hline Trust & $\begin{array}{l}\text { Assessment of physician's integrity, competence, and role as } \\
\text { patient's agent }\end{array}$ \\
\hline \multicolumn{2}{|l|}{ Structural feature of care } \\
\hline Organizational access & $\begin{array}{l}\text { Ability to get through to physician's office by telephone, to get } \\
\text { a medical appointment when sick, to obtain information by } \\
\text { telephone, punctuality of appointments, convenience of office } \\
\text { location, and convenience of office hours }\end{array}$ \\
\hline Financial access & $\begin{array}{l}\text { Assessment of amount of money patient pays for physician visits, } \\
\text { medication, and other prescribed treatments }\end{array}$ \\
\hline Visit-based continuity & $\begin{array}{l}\text { How often patient sees primary care physician (not an } \\
\text { assistant or partner) for routine check-ups and for appointments } \\
\text { when sick }\end{array}$ \\
\hline
\end{tabular}

delivered by large health plans and delivery systems over time (Murphy et al. 2001; Safran et al. 2002). Higher PCQ, as measured by the PCAS, has been linked to important patient outcomes such as adherence to physician's advice and improved health status (Safran et al. 1998).

The PCAS measures PCQ in the context of a specific physician-patient primary care relationship and references the entirety of that relationship (Safran et al. 1998). Nine summary scales (Table 1) cover two broad areas of PCQ: the patient-physician relationship (communication, interpersonal treatment, thoroughness of the physical exam, whole-person knowledge, preventive counseling, and trust) and structural/organizational features of care (organizational access, financial access, and visit-based continuity). Each scale has a range from 0 to 100 with higher scores indicating more of the underlying attribute. 


\section{Dependent Variable}

Each of the three outcomes were derived from the Addiction Severity Index (McLellan et al. 1992): (1) alcohol addiction severity using the alcohol composite score (ASI-alc), (2) drug addiction severity using the drug composite score (ASI-drug), and (3) any drug or heavy alcohol use (more than three drinks in a day) in the past 30 days. The latter variable will be referred to as "any substance use." ASI-alc and ASI-drug composite scores range from 0 to 1 , with higher scores indicating greater severity.

\section{Statistical Analysis}

We examined the relationship between higher PCQ and addiction outcomes by fitting separate multivariable longitudinal regression models for each outcome. Each regression model included a single PCAS scale as the main predictor variable. The unit of analysis was each study interview (i.e., observation). All study participants $(n=183)$ contributed to the analyses of any substance use ( $n=355$ observations). Only participants with alcohol as a first or second substance of choice $(n=117)$ contributed to the alcohol severity analyses ( $n=228$ observations). Likewise, only subjects with heroin or cocaine as a first or second drug of choice $(n=145)$ contributed to the drug severity analyses ( $n=284$ observations).

The longitudinal regression models accounted for the correlation from using repeated observations on the same subject. Continuous outcomes (ASIalc and ASI-drug) were analyzed using a general linear model for correlated data with an unstructured correlation matrix (Liang and Zeger 1986). For the dichotomous outcome of any substance use, generalized estimating equations (GEE) logistic regression models with empirical standard errors were used to analyze the data (Zeger, Liang, and Albert 1988). Each regression model also included the following covariates: age, gender, race/ethnicity, education less than 12 years (yes versus no), homelessness (any night in a shelter or street in the past 6 months); (Kertesz et al. 2005), health insurance, addiction severity (baseline alcohol and drug ASI scores at HELP study entry), HELP randomization group, time of addiction outcome assessment after HELP study entry $(12,18$, or 24 months), and interval between assessments of PCQ and addiction outcomes $(6,12$, or 18 months). No adjustments were made for multiple comparisons due to the exploratory nature of the analyses.

In order to assess whether PCQ was a predictor of subsequent addiction severity and substance use, models included addiction outcomes measured at the study interview after the PCQ assessment interview. As research interviews 
were scheduled approximately every 6 months over the 24-month study period, the interval between PCAS administration and the addiction assessment was 6 months if consecutive follow-up interviews were completed. However, if a participant missed a scheduled follow-up interview, then the substance abuse assessment at the next available interview was used (12 or 18 months after the assessment of PCQ). The interval between the PCQ assessment and addiction outcomes was 6 months for 269 (75.8 percent) of the 355 available observations; 12 months for 60 (16.9 percent); and 18 months for 26 (7.3 percent). A term for the interaction between each PCAS scale and the duration of time between PCQ assessment and addiction outcome assessment was included in initial models to assess whether the effect of PCQ depended upon the length of time between PCQ and addiction assessments. The interactions were not significant $(p>.1)$ and therefore not included in the final models.

Longitudinal regression results for addiction severity are presented as the mean difference in alcohol or drug severity associated with a standard deviation increase in PCAS score. Similarly, regression results for substance use are presented as the risk of substance use associated with a standard deviation increase in PCAS score. Reported p-values were two-tailed and considered statistically significant if $<.05$. All analyses were completed using SAS/STAT software, version 8.2 (SAS/STAT 1999).

\section{RESULTS}

\section{Study Subjects}

This study's analytic sample was derived from the HELP cohort. Of the 470 subjects in the HELP cohort, two died before follow-up and 400/468 (85 percent) completed at least one interview during the two-year follow-up period. Of the 400 subjects with follow-up, 253 (63 percent) reported initiating primary medical care after being discharged from the detoxification unit. As previously reported, women were more likely to link with primary care as well as those with recent episodic medical visits, family support for abstinence, and health insurance (Saitz et al. 2004). Recent incarceration decreased the likelihood of linkage. Ethnicity, recent addiction or mental health treatment utilization, addiction severity, health status, substance problem recognition, and perceived need for medical care did not affect linkage.

Among the 253 subjects who initiated primary care, 183 (72 percent) returned for a third interview (the "addiction interview") comprising our study sample. No significant differences were found between the 70 participants 
(70/253, 28 percent) who initiated primary care but were unavailable for the addiction interview and the study cohort in terms of age, gender, race/ethnicity, housing status, education level, alcohol abuse severity, or drug abuse severity.

The baseline sociodemographic and health characteristics of the study sample are displayed in Table 2 . A majority of study participants were male

Table 2: Demographic and Clinical Characteristics of Participants $(N=183)$ at Study Entry

\begin{tabular}{|c|c|}
\hline & $N(\%)$ \\
\hline Male & $124(68)$ \\
\hline \multicolumn{2}{|l|}{ Race/ethnicity } \\
\hline Black & $99(54)$ \\
\hline White & $51(28)$ \\
\hline Hispanic & $20(11)$ \\
\hline Less than 12 years education & $55(30)$ \\
\hline Homeless & $89(49)$ \\
\hline \multirow[t]{2}{*}{ Uninsured } & $110(60)$ \\
\hline & Mean $(\mathrm{SD})$ \\
\hline Age & $37(8)$ \\
\hline Physical health related quality of life* & $46(11)$ \\
\hline Mental health related quality of life $\mathrm{e}^{\dagger}$ & $32(12)$ \\
\hline Alcohol addiction severity ${ }^{\ddagger}$ & $0.45(0.35)$ \\
\hline Drug addiction severity ${ }^{\mathrm{s}}$ & $0.26(0.14)$ \\
\hline \multicolumn{2}{|l|}{ Primary Care Assessment Survey } \\
\hline \multicolumn{2}{|l|}{ Physician-patient interaction } \\
\hline Communication & $76(20)$ \\
\hline Interpersonal treatment & $74(21)$ \\
\hline Whole person knowledge & $54(24)$ \\
\hline Thoroughness of physical exam & $74(23)$ \\
\hline Trust & $73(17)$ \\
\hline Preventive counseling & $56(30)$ \\
\hline \multicolumn{2}{|l|}{ Structural features of care } \\
\hline Organization access & $62(21)$ \\
\hline Financial access & $81(24)$ \\
\hline Visit-based continuity & $83(24)$ \\
\hline
\end{tabular}

*Assessed with the Short-Form Health Survey (SF-36) Physical Component Summary (PCS), range $0-100$.

${ }^{\dagger}$ Assessed with the SF-36, Mental Component Summary (MCS), range 0-100.

${ }^{\ddagger}$ Addiction Severity Index (ASI) alcohol composite score range $0-1$ with higher scores indicating worse severity.

${ }^{\S}$ ASI drug composite score range $0-1$ with higher scores indicating worse severity.

"Primary Care Assessment Survey (PCAS) scales range from 0 to 100 points with higher scores indicating more of the underlying attribute. PCAS results from the first interview that a participant reported having a primary care provider are presented. 
(68 percent), nonwhite race/ethnicity (72 percent), and uninsured (60 percent). A substantial minority (30 percent) did not graduate from high school and about half were homeless. At baseline, the mean ASI-alc and mean ASI-drug scores were 0.45 and 0.26 , respectively. These scores are similar to those of individuals entering the public treatment system in Massachusetts (Smith and Larson 2003) but more severe than those in a clinical addiction treatment sample in an HMO in California (Weisner, McLellan, and Hunkeler 2000). The mean Short-Form Health Survey (SF-36) Physical Component Summary (PCS) and Mental Component Summary (MCS; Ware 1994) scores were 46 and 32 respectively (the mean MCS and PCS score for the U.S. population is 50 ; 89 percent of adults with MCS scores of 30-34 screens positive for depression). The lowest ranked measure of PCQ was whole-person knowledge and the highest was visit-based continuity.

\section{Multivariable Regression Results}

The 183 subjects contributed 355 observations to the longitudinal regression models. Table 3 presents the results of regression analyses examining the nine PCAS scales as independent predictors of alcohol and drug addiction severity. We were able to detect significant associations between PCQ and addiction outcomes most consistently in the alcohol addiction severity analyses. Higher

Table 3: Adjusted Mean Change in Alcohol and Drug Addiction Severity Associated with Higher Primary Care Quality

\begin{tabular}{lcccc}
\hline & $\begin{array}{c}\text { Alcohol Addiction } \\
\text { Severity Change* }\end{array}$ & $p$-Value & $\begin{array}{c}\text { Drug Addiction } \\
\text { Severity Change }\end{array}$ & $p$-Value \\
\hline Physician-patient interaction & & & & \\
$\quad$ Communication & $-\mathbf{0 . 0 6}$ & $\mathbf{. 0 0 1}$ & -0.005 & .51 \\
Interpersonal treatment & $-\mathbf{0 . 0 6}$ & $\mathbf{. 0 0 4}$ & -0.006 & .51 \\
Thoroughness of physical exam & $-\mathbf{0 . 0 5}$ & $\mathbf{. 0 0 5}$ & -0.0006 & .93 \\
Whole-person knowledge & $-\mathbf{0 . 0 6}$ & $\mathbf{. 0 0 1}$ & $-\mathbf{0 . 0 2}$ & .01 \\
Preventive counseling & -0.03 & .06 & -0.003 & .73 \\
Trust & $-\mathbf{0 . 0 4}$ & $\mathbf{. 0 2}$ & -0.007 & .40 \\
Structural features of care & & & & \\
Organizational access & $-\mathbf{0 . 0 6}$ & $\mathbf{. 0 0 2}$ & $-\mathbf{0 . 0 1}$ & $\mathbf{. 0 5}$ \\
Financial access & $-\mathbf{0 . 0 5}$ & $\mathbf{. 0 1}$ & -0.0002 & .97 \\
Visit-based continuity & $-\mathbf{0 . 0 3}$ & $\mathbf{. 0 5}$ & $-\mathbf{0 . 0 1}$ & $\mathbf{. 0 3}$ \\
\hline
\end{tabular}

*Subjects with alcohol as a first or second substance of choice $(n=115)$ contributed to alcohol addiction severity analyses ( $n=224$ observations).

${ }^{\S}$ Subjects with heroin or cocaine as a first or second drug of choice $(n=145)$ contributed to drug addiction severity analyses ( $n=280$ observations).

Statistically significant associations $(p<.05)$ in bold. 
scores on 8 of the 9 PCAS scales representing higher PCQ were associated with lower alcohol addiction severity at follow-up. Two measures of relationship quality (communication and whole person knowledge of the patient) were associated with the largest decrease in alcohol severity (ASI-alc -0.06 ); visitbased continuity was associated with the smallest decrease $(-0.03)$.

The results of drug severity (Table 3 ) and any substance use analyses (Table 4) were mixed. Higher scores on three PCAS scale scores (wholeperson knowledge, organizational access, and visit-based continuity) were associated with lower drug addiction severity: adjusted mean decrease in ASI-drug $=-0.01$ to -0.02 for each of the three PCAS scales. The other six PCAS scale scores were not significantly associated with drug addiction severity.

More whole-person knowledge and trust were significantly associated with lower odds of substance use (Table 4) (adjusted odds ratio, $[\mathrm{AOR}]=0.65$, 95 percent confidence interval $[95 \% \mathrm{CI}]=0.51,0.83$ and $\mathrm{AOR}=0.76,95$ percent $\mathrm{CI}=0.59,0.97$, respectively). Other PCAS scales did not significantly predict substance use.

To address the possibility of higher order effects of the HELP intervention, we tested for an interaction between HELP randomization group and

Table 4: Adjusted Odds Ratio of Any Substance Use at Follow-up Associated with Higher Primary Care Quality

Adjusted Odds Ratio

Primary Care Quality Attribute

(95\% Confidence Interval) of Any Substance Use*

$\begin{array}{ll}\text { Physician-patient interaction } & \\ \text { Communication } & 0.95(0.73,1.25) \\ \text { Interpersonal treatment } & 0.80(0.63,1.02) \\ \text { Thoroughness of physical exam } & 0.99(0.76,1.30) \\ \text { Whole person knowledge } & \mathbf{0 . 6 5}(\mathbf{0 . 5 1}, \mathbf{0 . 8 3}) \\ \text { Preventive counseling } & 0.91(0.74,1.13) \\ \text { Trust } & \mathbf{0 . 7 6}(\mathbf{0 . 5 9 , 0 . 9 7 )} \\ \text { Structural features of care } & \\ \text { Organizational access } & 0.86(0.68,1.09) \\ \text { Financial access } & 0.83(0.66,1.03) \\ \text { Visit-based continuity } & 0.94(0.76,1.17)\end{array}$

*Substance use is defined as any drug use (cocaine or heroin) or any alcohol intoxication ( $>3$ drinks on any occasion) in past the 30 days. Substance use was assessed 6 to 18 months after the primary care quality evaluation.

Results are reported as the adjusted odds ratio associated with a standard deviation increase in PCAS scale score (indicating more of the underlying attribute). Statistically significant associations $(p<.05)$ in bold. All study subjects $(n=183)$ contributed to analyses of substance use $(n=355$ observations). 
PCAS scale in each of the 27 main models. The interaction was only significant in the model examining organizational access and alcohol severity.

We explored whether primary care utilization affected the relationship between PCQ and addictions. In the main analyses, we did not adjust for primary care utilization because of the relatively low variability in the number of primary care visits. For 75 percent of the observations used in the analyses, patients reported between one and three primary care visits in the previous 6 months. Variability of the length of primary care relationships was also relatively narrow due to the fact that all study subjects initiated primary care during the study period. However, as primary care utilization is an important consideration, we included a covariate for the number of primary care visits (self-report) to the main models. Including this covariate did not alter our findings.

We also performed secondary analyses with covariates for exposure to substance abuse treatment (yes/no), AA participation (yes/no), and any mental health visit (yes/no), which again did not change the direction of the estimates or diminish the statistical significance of the results. However, in three of the models, the $p$-values increased: in the model examining visit-based continuity and alcohol addiction severity ( $p$-value increased from .05 to .06), and in the models examining visit-based continuity and organizational access predicting drug addiction severity ( $p$-values increased to .07). As the parameter estimates remained unchanged, higher $p$-values may have resulted from the addition of three more covariates to the models and diminished statistical power. Overall, the relationship between the quality of primary care and addiction outcomes did not appear to be mediated by these utilization variables.

\section{DISCUSSION}

In this cohort of adults recruited from a residential detoxification unit and prospectively assessed over a 24 -month study period, higher quality primary care across multiple domains was associated with lower addiction severity and odds of substance use. These associations did not appear to be mediated by variables previously identified in the literature to be related to health care quality (i.e., health insurance, gender, race/ethnicity), primary care utilization, or baseline addiction severity. Two key PCQ attributes reflecting the quality of primary care relationships-physicians' whole-person knowledge of the patient and patient trust-were significant predictors of lower alcohol severity and lower risk of substance use. 
Our findings align with those from previous studies that have demonstrated the importance of the physician-patient relationship in patient acceptance and receipt of preventive services (O'Malley et al. 2004), cancer screening measures (Safran et al. 1998; O'Malley and Forrest 2002), and depression treatment (O'Malley, Forrest, and Miranda 2003). Among HIV-infected individuals, trust and whole-person care has been linked with higher adherence to HIV medications (Schneider et al. 2004) as well as better physical and mental health functioning (Preau et al. 2004). Although the importance of interpersonal aspects of primary care has been reported in various populations, to our knowledge, this is the first study to examine their importance for patients with alcohol and drug use disorders.

In this study, whole-person knowledge emerged as the most consistent predictor of better addiction outcomes. Individualizing clinical decisions based upon the "contextual knowledge" of a patient's beliefs and values as well as responsibilities at work, home, or school (Weiner 2004) may have particular importance for individuals with addiction problems. It is notable that whole-person knowledge was one of the lowest ranked quality measure in this study, consistent with studies in other populations (Murphy et al. 2001). This suggests that careful attention to this aspect of primary care could be important when designing or evaluating programs that integrate addiction treatment with primary care.

It is important to note that the PCAS is not a measure of patient satisfaction but rather a well-validated measure of PCQ. The PCAS measures primary care in terms of a standard derived from the IOM's definition of primary care and may or may not relate to individual patient satisfaction. Other studies have measured PCQ in terms of delivery of specific services such as influenza vaccination or cervical screening. In light of our postulated mechanisms of higher quality primary care improving addictions, using the PCAS may have more relevance than using process of care measures.

Using this validated instrument to measure primary care, single standard deviation increases in PCQ scores were associated with a lower risk of subsequent substance use (i.e., 24-35 percent decrease in odds of any use) and moderate decreases in alcohol addiction severity (0.03-0.06 on scales ranging from 0 to 1). The reduction in ASI-alc scores demonstrated in this study are similar to the effect of two primary care visits versus none (ASI-alc $=-0.04$ ) found in a previous study (Saitz et al. 2005) but less than the effect of more intensive interventions such as case-managed residential care (Conrad et al. 1998) or work therapy for homeless veterans (ASI-alc $=-0.16)$ (Kashner et al. 2002). 
The clinical significance of the addiction differences in this study should be viewed in light of the fact that: (1) The magnitude of PCQ may be underestimated in this cohort with relatively new primary care relationships. Greater cumulative effects of higher quality primary care might be observed over a longer period of time. (2) The ability to robustly predict addiction outcomes with one exposure is rare. Since changes in addiction outcomes generally result from cumulative changes in the environment, it is not surprising that our findings were modest compared to more intensive interventions. We did not expect to find large differences in addiction severity among these patients, all of whom had initiated substance abuse treatment and were receiving primary care. As there is renewed interest in providing primary care to individuals with addictions, ensuring that core features of primary care exist in their primary care relationships might augment the postulated addiction benefits by the estimates found in this study.

While all of the effects of PCQ were in the hypothesized direction, we were unable to detect associations between attributes of PCQ and drug addiction severity. Despite adjusted analyses, the impact of the quality of primary care relationships initiated over a relatively short period of time may have been difficult to isolate in this cohort with significant homelessness and poverty. As drug dependence is more difficult to treat than alcohol dependence without adequate pharmacotherapy, the effect of primary care on addiction outcomes may have been overwhelmed without first reducing system-level barriers to opioid agonist therapy. Additionally, the prevalence of comorbid psychiatric conditions is generally higher in samples with drug addiction than those with alcohol addiction (Grant et al. 2004). Thus, detecting the effect of differences in PCQ may have been more difficult without addressing psychiatric comorbidities in patients with drug use disorders.

Several limitations should be considered when interpreting the study findings. The major limitation is the potential confounding resulting from this study's observational design. We cannot exclude the possibility that addiction severity was a determinant of the quality of primary care rather than PCQ predicting addiction outcomes. We attempted to minimize this possibility by: (1) using a lagged analysis, i.e., assessing the addiction severity of an individual at least 6 months after the receipt of primary care services; (2) including wellestablished determinants of PCQ, specifically, race/ethnicity, gender, and health insurance in the analyses; and (3) adjusting for baseline addiction severity. Still, unmeasured factors may have influenced which participants received better primary care and mediated addiction outcomes. A propensity 
analysis might have better addressed this limitation, however, this study's sample size could not support this approach.

Another limitation relates to the fact these data were primarily collected for a randomized trial of an intervention to link adults to primary care. It is unlikely, however, that the HELP intervention confounded our results since previous analyses demonstrated that the HELP intervention was not associated with addiction benefit. In addition, we included HELP randomization group in the models and did not find significant interactions between PCAS scores and HELP randomization group (except for the alcohol model with organizational access). However, we acknowledge that the impact of primary care on addiction outcomes may have been evident in individuals who were "primed" by the HELP intervention to be responsive to the effects of primary care.

Finally, generalizability of the study's findings is another limitation. It is important to note that these data reflect a single urban adult population with substantial social and economic problems as well as alcohol and drug use disorders severe enough to require detoxification admission. The sample is not representative of patients with substance use disorders found in primary care, who may have less severe and persistent addiction trajectories. Hence, it is unclear whether our findings would be applicable to patients with less severe substance use disorders and these findings should be examined in other cohorts. Regardless of whether these findings are applicable to other patients in primary care, the population that we studied is an important one as this cohort is representative of many clinical samples in public-funded treatment settings (Smith and Larson 2003).

This study's findings are relevant to recent efforts to expand primary care's involvement in the management of addictive disorders. Financial and structural integration between substance abuse services and primary medical care have been proposed to increase coordination of traditionally separate systems of care (National Council for Community Behaviorial Healthcare 2003; New Freedom Commission on Mental Health 2003). As policy-makers move forward with increasing primary care's role in managing substance use disorders, our study suggests that certain core components of primary care, particularly the ability to provide whole-person care, are important to realizing the postulated benefits of increasing access to primary care.

In summary, multiple features of PCQ were associated with lower addiction severity and risk of future substance use. Our findings suggest that enhancing primary care's ability to deliver patient-centered, comprehensive, longitudinal care may have a beneficial impact on addiction outcomes. 


\section{ACKNOWLEDGMENTS}

This work was supported by a grant from the National Institute on Alcohol Abuse and Alcoholism (RO1-AA10870) and the National Institute on Drug Abuse (RO1-DA10019) (R25-DA13582). Support for Theresa Kim came from the National Institute Drug Abuse (R25-DA13582). This work was also supported, in part, by the Boston University General Clinical Research Center from the National Center for Research Resources (MO1-RR00533).

\section{REFERENCES}

Background Paper: Behavioral Health/Primary Care Integration Models, Competencies, and Infrastructure. 2003. National Council for Community Behavioral Healthcare.

Campbell, S., M. Roland, and D. Wilkin. 2001. "Primary Care Groups: Improving the Quality of Care through Clinical Governance." British Medical Journal 322: $1580-2$.

Conrad, K. J., C. I. Hultman, A. R. Pope, J. S. Lyons, W. C. Baxter, and A. N. Daghestani. 1998. "Case Managed Residential Care for Homeless Addicted Veterans. Results of a True Experiment." Medical Care 36: 40-53.

De, A. I., J. H. Samet, and R. Saitz. 2004. "Burden of Medical Illness in Drug- and Alcohol-Dependent Persons without Primary Care." American Journal of Addiction 13: 33-45.

Fiellin, D. A., and P. G. O'Connor. 2002. "New Federal Initiatives to Enhance the Medical Treatment of Opioid Dependence." Annals of Internal Medicine 137: 68892.

Friedmann, P. D., Z. Zhang, J. Hendrickson, M. D. Stein, and D. R. Gerstein. 2003. "Effect of Primary Medical Care on Addiction and Medical Severity in Substance Abuse Treatment Programs." Journal of General Internal Medicine 18: 1-8.

Grant, B. F., F. S. Stinson, D. A. Dawson, S. P. Chou, M. C. Dufour, W. Compton, R. P. Pickering, and K. Kaplan. 2004. "Prevalence and Co-occurrence of Substance Use Disorders and Independent Mood and Anxiety Disorders: Results from The National Epidemiologic Survey on Alcohol and Related Conditions." Archives of General Psychiatry 61: 807-16.

Institute of Medicine. 1996. Primary Care: America's Health in a New Era. Washington, DC: National Academy Press.

Johnson, J. G., R. L. Spitzer, J. B. Williams, K. Kroenke, M. Linzer, D. Brody, F. deGruy, and S. Hahn. 1995. "Psychiatric Comorbidity, Health Status, and Functional Impairment Associated with Alcohol Abuse and Dependence in Primary Care Patients: Findings of the PRIME MD-1000 Study." Journal of Consulting and Clinical Psychology 63: 133-40.

Kashner, T. M., R. Rosenheck, A. B. Campinell, A. Suris, R. Crandall, N. J. Garfield, P. Lapve, K. Prycz, T. Soyka, and A. Wicker. 2002. "Impact of Work Therapy on 
Health Status among Homeless, Substance-Dependent Veterans: A Randomized Controlled Trial." Archives of General Psychiatry 59: 938-44.

Kertesz, S. G., M. J. Larson, N. J. Horton, M. Winter, R. Saitz, and J. H. Samet. 2005. "Homeless Chronicity and Health-Related Quality of Life Trajectories among Adults with Addictions." Medical Care 43: 574-85.

Kroenke, K. 2004. “The Many C's of Primary Care.” Journal of General Internal Medicine 19: 708-9.

Liang, K., and S. L. Zeger. 1986. "Longitudinal Data Analysis Using Generalized Linear Models." Biometrika 73: 13-22.

Maisto, S. A., and R. Saitz. 2003. "Alcohol Use Disorders: Screening and Diagnosis." American Journal of Addiction 12 (suppl 1): S12-25.

McLellan, A. T., H. Kushner, D. Metzger, R. Peters, I. Smith, and G. Grissom. 1992. "The Fifth Edition of the Addiction Severity Index." Journal of Substance Abuse Treatment 9: 199-213.

McLellan, A. T., D. C. Lewis, C. P. O’Brien, and H. D. Kleber. 2000. “Drug Dependence, a Chronic Medical Illness: Implications for Treatment, Insurance, and Outcomes Evaluation.” Journal of the American Medical Association 284: 1689-95.

Mertens, J. R., Y. W. Lu, S. Parthasarathy, C. Moore, and C. M. Weisner. 2003. "Medical and Psychiatric Conditions of Alcohol and Drug Treatment Patients in an HMO: Comparison with Matched Controls." Archives of Internal Medicine 163: 2511-17.

Murphy, J., H. Chang, J. E. Montgomery, W. H. Rogers, and D. G. Safran. 2001. "The Quality of Physician-Patient Relationships. Patients' Experiences 1996-1999.” Journal of Family Practice 50: 123-9.

New Freedom Commission on Mental Health. 2003. Achieving the Promise: Transforming Mental Healthcare in America. Executive Summary. SMA-03-3831. Rockville, MD: DHHS.

O’Malley, A. S., and C. B. Forrest. 2002. "Beyond The Examination Room: Primary Care Performance and the Patient-Physician Relationship for Low-Income Women." Journal of General Internal Medicine 17: 66-74.

O’Malley, A. S., C. B. Forrest, and J. Miranda. 2003. "Primary Care Attributes and Care for Depression among Low-Income African American Women.” American Journal of Public Health 93: 1328-34.

O'Malley, A. S., V. B. Sheppard, M. Schwartz, and J. Mandelblatt. 2004. "The Role of Trust in Use of Preventive Services among Low-Income African-American Women." Preventive Medicine 38: 777-85.

Preau, M., C. Leport, D. Salmon-Ceron, P. Carrieri, H. Portier, G. Chene, B. Spire, P. Chouter, F. Raffi, and M. Morin. 2004. "Health-Related Quality of Life and Patient-Provider Relationships in HIV-Infected Patients during the First Three Years after Starting PI-Containing Antiretroviral Treatment." AIDS Care 16: 649-61.

Safran, D. G. 2003. "Defining the Future of Primary Care: What Can We Learn from Patients?" Annals of Internal Medicine 138: 248-55.

Safran, D. G., M. Kosinski, A. R. Tarlov, W. H. Rogers, D. H. Taira, N. Lieberman, and J.E. Ware. 1998. "The Primary Care Assessment Survey: Tests of Data Quality and Measurement Performance." Medical Care 36: 728-39. 
Safran, D. G., D. A. Taira, W. H. Rogers, M. Kosinski, J. E. Ware, and A. R. Tarlov. 1998. "Linking Primary Care Performance to Outcomes of Care." Journal of Family Practice 47: 213-20.

Safran, D. G., I. B. Wilson, W. H. Rogers, J. E. Montgomery, and H. Chang. 2002. "Primary Care Quality in The Medicare Program: Comparing the Performance of Medicare Health Maintenance Organizations and Traditional Fee-for-Service Medicare." Archives of Internal Medicine 162: 757-65.

Saitz, R., N. J. Horton, M. J. Larson, M. Winter, and J. H. Samet. 2005. "Primary Medical Care and Reductions in Addiction Severity: A Prospective Cohort Study." Addiction 100: 70-8.

Saitz, R., N. J. Horton, L. M. Sullivan, M. A. Moskowitz, and J. H. Samet. 2003. "Addressing Alcohol Problems in Primary Care: A Cluster Randomized, Controlled Trial of a Systems Intervention. The Screening and Intervention in Primary Care (SIP) Study." Annals of Internal Medicine 138: 372-82.

Saitz, R., M. J. Larson, N. J. Horton, M. Winter, and J. H. Samet. 2004. "Linkage with Primary Medical Care in a Prospective Cohort of Adults with Addictions in Inpatient Detoxification: Room for Improvement." Health Services Research 39: 587-606.

Saitz, R., K. P. Mulvey, A. Plough, and J. H. Samet. 1997. "Physician Unawareness of Serious Substance Abuse." American Journal of Drug and Alcohol Abuse 23: 343-54.

Saitz, R., L. M. Sullivan, and J. H. Samet. 2000. "Training Community-Based Clinicians in Screening and Brief Intervention for Substance Abuse Problems: Translating Evidence into Practice.” Substance Abuse 21: 21-31.

Samet, J. H., M. J. Larson, N. J. Horton, K. Doyle, M. Winter, and R. Saitz. 2003. "Linking Alcohol- and Drug-Dependent Adults to Primary Medical Care: A Randomized Controlled Trial of a Multi-Disciplinary Health Intervention in a Detoxification Unit." Addiction 98: 509-16.

SAS/STAT. 1999. Version 8.2 of the SAS System for Windowes. (8.2). 2001. Cary, NC: SAS Institute Inc.

Schneider, J., S. H. Kaplan, S. Greenfield, W. Li, and I. B. Wilson. 2004. "Better Physician-Patient Relationships Are Associated with Higher Reported Adherence to Antiretroviral Therapy in Patients with HIV Infection.” Journal of General Internal Medicine 19: 1096-103.

Smith, K. W., and M.J. Larson. 2003. "Quality of Life Assessments by Adult Substance Abusers Receiving Publicly Funded Treatment in Massachusetts.” American Journal of Drug and Alcohol Abuse 29: 323-35.

Starfield, B., C. Cassady, J. Nanda, C. B. Forrest, and R. Berk. 1998. "Consumer Experiences and Provider Perceptions of the Quality of Primary Care: Implications for Managed Care." Journal of Family Practice 46: 216-26.

Ware, J. E. 1994. SF-36 Physical and Mental Health Summary Scales: A User's Manual. Boston: The Health Institute.

Weiner, S. J. 2004. "Contextualizing Medical Decisions to Individualize Care: Lessons from the Qualitative Sciences.” Journal of General Internal Medicine 19: 281-85. 
Weisner, C., and H. Matzger. 2003. "Missed Opportunities in Addressing Drinking Behavior in Medical and Mental Health Services." Alcoholism Clinical and Experimental Research 27: 1132-41.

Weisner, C., A. T. McLellan, and E. M. Hunkeler. 2000. “Addiction Severity Index Data from General Membership and Treatment Samples of HMO Members. One Case of Norming the ASI." Journal of Substance Abuse Treatment 19: 103-9.

Weisner, C., J. Mertens, S. Parthasarathy, C. Moore, and Y. Lu. 2001. "Integrating Primary Medical Care with Addiction Treatment: A Randomized Controlled Trial." Journal of the American Medical Association 286: 1715-23.

Welte, J. W., P. Perry, R. Longabaugh, and P. R. Clifford. 1998. "An Outcome Evaluation of a Hospital-Based Early Intervention Program.” Addiction 93: 573-81.

Willenbring, M. L., and D. H. Olson. 1999. "A Randomized Trial of Integrated Outpatient Treatment for Medically Ill Alcoholic Men.” Archives of Internal Medicine 159: 1946-52.

Zeger, S. L., K. Y. Liang, and P. S. Albert. 1988. "Models for Longitudinal Data: A Generalized Estimating Equation Approach.” Biometrics 44: 1049-60. 